\title{
Orientaciones de aculturación, estrés de aculturación y bienestar psicológico en inmigrantes latinoamericanos en Santiago de Chile
}

\author{
María José Mera-Lemp; Gonzalo Martínez-Zelaya; Aracely Orellana; Vanessa Smith-Castro
}

How to cite this article:

Mera-Lemp, M.J., Martínez-Zelaya, G., Orellana, A. \& Smith-Castro, V. (2020). Acculturation orientations, acculturative stress and psychological well-being on Latin American immigrants settled in Santiago, Chile. Acta Colombiana de Psicología, 23(1), 216-230. doi: http://www.doi.org/10.14718/ACP.2020.23.1.11

Recibido, septiembre 26/2018; Concepto de evaluación, abril 1/2019; Aceptado, junio 26/2019

\author{
María José Mera-Lemp \\ Universidad Alberto Hurtado, Santiago, Chile. \\ ORCID: https://orcid.org/0000-0001-5763-6913 \\ Gonzalo Martínez-Zelaya* \\ Universidad Viña del Mar, Viña del Mar, Chile \\ ORCID: https://orcid.org/000-0002-9848-3666 \\ Aracely Orellana \\ Fundación Scalabrini (Chile), Scalabrini International Migration Network (SIMN) \\ ORCID: https://orcid.org/000-0002-0293-6182 \\ Vanessa Smith-Castro \\ Universidad de Costa Rica, San Pedro, Costa Rica. \\ ORCID: https://orcid.org/0000-0001-6348-4223
}

\begin{abstract}
Resumen
En Chile, el incremento del flujo migratorio desde América Latina ha llamado la atención con respecto a los procesos de ajuste psicológico y sociocultural de los nuevos inmigrantes. Por tanto, con este trabajo se busca (a) establecer relaciones entre las orientaciones de aculturación, el estrés aculturativo y el bienestar psicológico, (b) identificar perfiles según las preferencias de aculturación, y (c) determinar la existencia de diferencias significativas en el estrés y el bienestar, de acuerdo con estas tipologías. Para esto, se realizó un estudio de tipo correlacional con una muestra de $(\mathrm{N}=194)$ inmigrantes latinoamericanos con edades entre los 18 y los 67 años $(\mathrm{M}=34.77$; $\mathrm{DE}=10.181)$, donde se evaluaron las orientaciones de aculturación, el estrés por aculturación y el bienestar psicológico. Los resultados indican que el predominio de la integración es más salutogénico que la tendencia a la separación cultural como orientación preeminente, lo que facilita un mejor afrontamiento de los estresores del proceso migratorio. Asimismo, se encontraron tres perfiles aculturativos -individualista moderado, integracionista y separatista - y la comparación entre perfiles arroja que cuando la integración es más saliente, el bienestar es mayor que en presencia del individualismo como orientación predominante. Este trabajo aporta evidencia con respecto al individualismo como orientación de aculturación escasamente considerada en la literatura, cuya posible influencia negativa puede relacionarse con la pertenencia grupal como vía para acceder a recursos que aportan a la adaptación al nuevo contexto.

Palabras clave: aculturación, migración, estrés aculturativo, bienestar psicológico.
\end{abstract}

\footnotetext{
Escuela de Ciencias Jurídicas y Sociales, Universidad Viña del Mar, Los Fresnos 91, Viña del Mar, Chile. Tel: +56 322462400 , gonzalo.martinez@uvm.cl
}

Este trabajo contó con el financiamiento del proyecto CONICYT/FONDECYT postdoctoral n. ${ }^{\circ} 3180774$. 


\title{
Acculturation orientations, acculturative stress and psychological well-being on Latin American immigrants settled in Santiago, Chile
}

Abstract

\begin{abstract}
In Chile, the significant increase of migratory flows from Latin America has drawn attention to the psychological and sociocultural adjustment processes of new immigrants. This paper seeks to (i) establish relationships between acculturation orientations, acculturative stress and psychological well-being, (ii) identify profiles according to acculturation preferences, and (iii) determine the existence of significant differences in stress and well-being between these profiles. This correlational study was conducted with a sample of $(\mathrm{N}=194)$ Latin American immigrants with ages between 18 and 67 years $(\mathrm{M}=34.77 ; \mathrm{DE}=10.181)$, where acculturation orientations, acculturation stress and psychological well-being were evaluated. Results indicate that the predominance of integration is more salutogenic than the tendency to cultural separation as a preeminent orientation, facilitating a better coping with the stressors of the migratory process. Three acculturative profiles were found: moderate individualist, integrationist and separatist. The comparison between profiles shows that when integration is predominant, well-being is greater than in the presence of individualism as the principal orientation. This study provides evidence regarding individualism as an acculturation orientation scarcely considered in the literature, whose possible negative influence can be related to group membership as a way to access resources that collaborate with adaptation to the new context.

Key words: acculturation, migration, acculturative stress, psychological well-being.
\end{abstract}

\section{Introducción}

En Chile, las transformaciones sociales y económicas experimentadas en las últimas décadas han configurado un nuevo patrón migratorio caracterizado por un importante incremento en el número de inmigrantes, una concentración de la población extranjera en la Región Metropolitana de Santiago, y un sostenido aumento en la llegada de personas desde diversos países de América Latina (Stefoni, 2011), quienes representan el $81.3 \%$ del total de inmigrantes en el país (Instituto Nacional de Estadísticas, 2018).

Con respecto a este fenómeno, algunos estudios realizados en el país (Cárdenas, 2006; González, Sirlopú \& Kessler, 2010; Mera, Martínez-Zelaya, Bilbao \& Garrido, 2017; Sirlopú \& van Oudenhoven, 2013; Stefoni, 2011; Valenzuela et al., 2018) han reportado la existencia de actitudes negativas por parte de la población chilena hacia la población inmigrante latinoamericana. Así también, la inexistencia hasta la fecha de una política pública integral que garantice y facilite el ejercicio de derechos por parte de la población extranjera, especialmente en cuanto a la obtención de permisos de residencia, así como el acceso a la salud, a la educación, a la vivienda y al trabajo ha sido señalada como una importante barrera para su inclusión y la consecución de los proyectos migratorios (Galaz, Poblete \& Frías, 2017; Stefoni, 2011; Valenzuela et al, 2018).

Asimismo, las dificultades relacionadas con la regularización de la situación jurídico-administrativa a la que se asocia el acceso a diversos servicios y programas sociales del Estado (Galaz et al., 2017) supone que en la cotidianeidad las personas inmigrantes deban afrontar múltiples estresores ambientales y sociales, a los que se suma la gestión de las diferencias culturales (Urzúa, Basabe, Pizarro \& Ferrer, 2017) y el bajo estatus derivado de la desigualdad en el reconocimiento y ejercicio de derechos (Bourhis, Moïse, Perreault \& Senécal, 1997; Galaz et al., 2017). La presencia de estas tensiones entre inmigrantes y autóctonos es relevante, pues el contacto intergrupal constituye un elemento crítico que moviliza cambios identitarios, actitudinales y conductuales bidireccionales que han sido conceptualizados como procesos de aculturación.

Respecto a esto, el enfoque bidimensional de la aculturación, descrito por Berry (2005), asume que la relación con la sociedad receptora plantea para los inmigrantes dos cuestiones básicas: en qué medida su identidad cultural de origen es valiosa y desean mantenerla; $y$, al mismo tiempo, en qué grado desean incorporar la cultura de la sociedad mayoritaria (Berry, 2005; Bourhis et al., 1997). Además, se considera que las actitudes hacia ambas culturas no son mutuamente excluyentes, y que pueden variar de forma independiente. Adicional a esto, Berry (2005) diferencia cuatro orientaciones de aculturación: (a) la integración o biculturalismo, que supone el mantenimiento de la identidad cultural del grupo de pertenencia y la adopción de la cultura de la sociedad receptora; (b) la asimilación, una fuerte orientación hacia la cultura del país de recepción y el abandono de la cultural de origen; (c) la separación, que conlleva el mantenimiento de fuertes vínculos con el grupo de pertenencia y una actitud negativa hacia la cultura de recepción; y (d) la marginalización, que refiere a una actitud negativa hacia ambas culturas.

Adicionalmente, Bourhis et al. (1997) proponen que, en el contexto de las migraciones voluntarias y motivadas 
por el deseo de mejorar las condiciones de vida personales y familiares, los inmigrantes pueden desarrollar una quinta orientación, el individualismo, que consiste en la identificación de sí mismos como individuos, más que como miembros de grupos, y representa una estrategia de movilidad social personal. Esto supone un mayor involucramiento con el logro de las propias metas que con la mantención de su cultura o la adopción de la cultura del país receptor.

Cabe mencionar que las orientaciones de aculturación son desplegadas por los individuos ante un conflicto entre dos culturas que se actualiza y resuelve en la interacción cotidiana con la sociedad de recepción, por lo que, aunque ciertas preferencias aculturativas pueden tener mayor saliencia, su funcionamiento es dinámico e interrelacionado (Barrete, Bourhis, Personnaz \& Personnaz, 2004).

En el nivel individual, estos procesos implican una reorganización emocional y sociocognitiva que pueden provocar malestar psicológico, en especial cuando las condiciones contextuales son adversas (Ochoa, Vicente \& Lozano, 2005). Ahora bien, el estrés asociado con demandas de adaptación a la nueva cultura que exceden las capacidades de las personas para afrontarlas se denomina estrés por aculturación (Ugalde-Watson, Smith-Castro, Moreno-Salas \& Rodríguez-García, 2011; Williams \& Berry, 1991), y se relaciona tanto con los desafíos culturales e identitarios propios de la migración, como con aspectos relativos a la preparación del traslado, la regularización de la situación jurídica en el país de recepción y las dificultades económicas (Ugalde-Watson et al., 2011). Estos estresores se caracterizan por su intensidad, su multiplicidad y su presencia sostenida, en ocasiones, por largos periodos de tiempo, lo que incrementa su probabilidad de afectar la salud mental de las personas inmigrantes (Achotegui, 2006).

Estudios previos han proporcionado evidencia con respecto a la relación entre los estilos de aculturación con el estrés y la salud mental percibida por los inmigrantes. Por ejemplo, la orientación hacia la sociedad de recepción, y, en específico, la tendencia a la integración, han sido identificadas como las más satulogénicas en muestras transculturales (Basabe, Páez, Aierdi \& Jiménez-Aristizabal, 2009; Chen, Benet-Martínez \& Bond, 2008; Kuo, 2014; Torres, Driscoll \& Voell, 2012; Urzúa, Boudon \& Caqueo-Urizar, 2017; Yáñez \& Cárdenas, 2010). Frente a esto último, un estudio metaanalítico realizado por Yoon et al. (2013) con 325 investigaciones en este ámbito confirmó esta relación y, además, corroboró el vínculo negativo de la marginalización con diferentes indicadores de salud mental.

Con respecto a la asimilación y la separación, no se ha encontrado evidencia concluyente sobre la naturaleza de su relación con el estrés y la salud mental. Si bien Yoon et al. (2013) encontraron asociaciones con menor estrés y mayor salud mental - aunque en menor medida que con la integración-, existen diversos estudios que han reportado lo contrario, estableciendo relaciones positivas entre la presencia de sintomatología psicopatológica con la asimilación (Elgorriaga, 2011; Urzúa et al., 2017) y la separación (Basabe et al, 2009; Berry \& Sabatier, 2010; Elgorriaga, 2011; Navas et al., 2004; Urzúa, Heredia \& Caqueo-Urizar, 2016).

Por otra parte, en cuanto al individualismo como orientación de aculturación y su relación con el estrés y la salud mental, la evidencia disponible es escasa; sin embargo, de manera muy aislada, en algunas investigaciones se ha informado de relaciones positivas con síntomas depresivos graves, como con la ideación suicida (van Leeuwen, Rodgers, Bui, Pirlot \& Chabrol, 2014; van Leeuwen, Rodgers, Régner $\&$ Chabrol, 2010). En Chile, de igual forma, en un estudio reciente con inmigrantes latinoamericanos, Urzúa et al. (2017) encontraron que el despliegue de estrategias de afrontamiento de individualización se asoció a un mayor estrés aculturativo.

Por lo general, los estudios que abordan la relación entre la aculturación y el bienestar en inmigrantes se enmarcan en la tradición hedónica y tienen en consideración aspectos como las emociones en el proceso migratorio y la satisfacción con la vida. Esta línea de investigación ha proporcionado evidencia consistente con la existencia de relaciones entre el bienestar y las características sociodemográficas y culturales de los inmigrantes, sus orientaciones de aculturación, y el tipo de relación que sostienen con las sociedades receptoras (Bobowick, Basabe \& Páez, 2014; Urzúa, Sánchez, Paniagua, Igor \& Castro, 2015).

Recientemente, al estudio del ajuste de los inmigrantes se ha incorporado el enfoque de la eudaemonia (Bobowick et al., 2014; Silva et al., 2016; Urzúa et al., 2015), que atiende al bienestar psicosocial. Desde esta perspectiva (Ryff, 1989), el bienestar psicológico es definido como un constructo multidimensional que involucra el desarrollo de las capacidades y el crecimiento personal, la autoaceptación, el sentimiento de control sobre el entorno y el establecimiento de relaciones positivas con los otros. Esta aproximación resulta particularmente interesante en el contexto de la inmigración, pues pone de relevancia aspectos y experiencias de la vida cotidiana que pueden ser afectadas durante el proceso migratorio y que pueden incidir en la salud mental de las personas migrantes (Bobowik et al., 2014; Silva et al., 2016). Aunque los estudios sobre su relación con la aculturación son incipientes, en ellos se ha evidenciado claramente la existencia de asociaciones entre ambas variables. Por ejemplo, en población inmigrante en el Norte 
de Chile se ha encontrado que la integración se relaciona positivamente con el bienestar psicológico, mientras que la asimilación se asocia negativamente bienestar (Silva et al., 2016; Yáñez \& Cárdenas, 2010).

Con base en los antecedentes presentados, en la presente investigación se plantean tres objetivos: el primero, determinar la existencia de relaciones entre las orientaciones de aculturación, el estrés aculturativo y el bienestar psicológico de una muestra de inmigrantes latinoamericanos en Santiago de Chile; el segundo, de carácter exploratorio, establecer tipologías a partir de la agrupación de los sujetos en torno a sus preferencias de aculturación; y, el tercero, determinar la existencia de diferencias significativa en estrés y bienestar según las tipologías de aculturación.

En cuanto a las hipótesis, se espera que la orientación hacia la integración cultural se relacione positivamente con el bienestar psicológico y de manera negativa con el estrés de aculturación. Por el contrario, se espera que la marginalización y el individualismo se relacionen negativamente con el bienestar y positivamente con el estrés aculturativo. Adicionalmente, si bien la literatura presenta resultados contradictorios con respecto a la relación entre la asimilación y la segregación, así como con el bienestar, en este estudio se espera que ambas orientaciones se relacionen negativamente con el bienestar y positivamente con el estrés. $\mathrm{Y}$, por último, con respecto a los perfiles de aculturación, se espera que el predominio del integracionismo se asocie a un mayor bienestar y a un menor estrés que en grupos en los que predominen las demás orientaciones de aculturación.

\section{Método}

\section{Diseño}

El presente estudio es de tipo no experimental, transversal y de corte cuantitativo, ya que busca responder con respecto a tendencias generales en la población inmigrante latinoamericana de Santiago de Chile. El alcance de esta investigación es de tipo comparativo relacional (Hernández, Baptista \& Fernández, 2010).

\section{Participantes}

La muestra estuvo compuesta por 194 inmigrantes latinoamericanos, hombres (50\%) y mujeres (50\%), asentados en Santiago de Chile, de entre 18 y 67 años $(\mathrm{M}=34.77$; $D E=10.181)$. El $35.6 \%$ contaba con estudios universitarios, el $13.9 \%$ con estudios de posgrado, el $24.2 \%$ con estudios técnicos, el $23.2 \%$ con estudios secundarios y el $3.1 \%$ había realizado solo estudios primarios. La mayoría procedía de países como Colombia (31.5\%), Venezuela (28.9\%), Perú (13.4\%), Bolivia (6.2\%), Ecuador (5.2\%) y México (5.2\%), mientras que el resto era proveniente de República Dominicana (2.6\%), Brasil (2.1\%), Haití (1.5\%), El Salvador (1\%), Paraguay (1\%), Costa Rica $(0.5 \%)$, Cuba ( $0.5 \%)$ y Argentina $(0.5 \%)$. Los participantes tuvieron una permanencia media en Chile de 19.9 meses $(D E=31.3 ;$ rango $=2$ a 240 meses $)$. Se excluyeron de esta muestra a las personas con estatus de refugiado.

\section{Variables e instrumentos}

Variables sociodemográficas. Se administró un cuestionario en el que se registró la edad, el sexo, la nacionalidad, el nivel educativo y el tiempo de estancia en Chile de los participantes.

Orientaciones de aculturación. Se utilizó la subescala "Mantención cultural" de la Immigrant Acculturation Scale (Bourhis \& Barrette, 2006), traducida al castellano y validada en población latinoamericana por Azurmendi y Larrañaga (2008), que consta de cinco ítems tipo Likert con siete opciones de respuesta $(1=$ "totalmente en desacuerdo", 7 = "totalmente de acuerdo") para medir el grado en que las personas inmigrantes se orientan hacia la integración (“deseo mantener la cultura de mi país de origen y además adoptar aspectos de la cultura chilena”), separación (“deseo mantener la cultura de mi país de origen, en vez de adoptar la cultura chilena"), individualismo ("me preocupan poco la cultura de mi país de origen y la cultura chilena, pues son mis necesidades personales y mis propias aspiraciones lo que realmente importa para mí"), asimilación ("deseo dejar la cultura de mi país de origen, para adoptar la cultura chilena"), y marginalización cultural ("no quiero mantener la cultura de mi país de origen, ni adoptar la cultura chilena, pues me siento incómodo/a con ambas culturas").

Estrés por aculturación. Se utilizó la Escala de Estrés de Aculturación de Ugalde-Watson et al. (2011). El instrumento original consta de 33 ítems tipo Likert con cinco opciones de respuesta ( 1 = "nada estresante", $5=$ "muy estresante") que miden el grado de estrés percibido en cuatro dimensiones del proceso migratorio: adaptación cultural y social a la sociedad receptora, preocupaciones económicas, preparativos para salir del país de origen, y el estrés por la adaptación al estatus jurídico de refugiado (“¿qué tan estresante, tenso o angustiante le resultó o cree que le va a resultar ciertas situaciones?... Estabilizarse económicamente"). 
Aunque no se han encontrado aplicaciones de esta escala en el contexto chileno, estudios previos en población inmigrante latinoamericana dan cuenta de propiedades psicométricas adecuadas. Por ejemplo, Ugalde-Watson et al. (2011) en una muestra de inmigrantes colombianos en Costa Rica reportaron adecuados alfas de Cronbach para cada subescala: adaptación a la sociedad receptora $(\alpha=.91)$, adaptación al estatuto jurídico de refugiado $(\alpha=.83)$, preocupaciones socioeconómicas $(\alpha=.81)$, y preparación para la emigración $(\alpha=.77)$. En este estudio, dado que no se trabajó con población refugiada, se adecuó la redacción de algunos ítems a la condición de inmigrante, en reemplazo de la condición de refugiados. En la muestra estudiada en esta investigación, la escala contó con una fiabilidad general de alfa .92 y omega .92. La fiabilidad para cada dimensión también fue adecuada: adaptación a la sociedad receptora $(\alpha=.92)$, adaptación al estatuto jurídico $(\alpha=.73)$, preocupaciones socioeconómicas $(\alpha=.87)$, y preparación para la emigración $(\alpha=.85)$.

Bienestar psicológico. Se utilizó la Escala de Bienestar Psicológico de Ryff (1989) adaptada al español por Díaz et al. (2006). La adaptación española consta de un total de 29 ítems con formato de respuesta tipo Likert que oscila entre 1 ("totalmente en desacuerdo") y 5 ("totalmente de acuerdo"), con afirmaciones como "en general, me siento seguro y positivo conmigo mismo".

Adicionalmente, el instrumento considera la medición de seis dimensiones, cuya fiabilidad en este estudio es aceptable: autoaceptación $(\alpha=.63)$, relaciones positivas con los otros $(\alpha=.61)$, autonomía $(\alpha=.62)$, dominio del entorno $(\alpha=.65)$, propósito en la vida $(\alpha=.81)$ y crecimiento personal $(\alpha=.67)$. La fiabilidad general obtenida fue de $\alpha=.88$. En otros estudios con población inmigrante latinoamericana en Chile, este instrumento también ha presentado propiedades psicométricas adecuadas. Así, por ejemplo, Yáñez y Cárdenas (2010) reportaron una fiabilidad general de $\alpha=.77$ para esta escala.

\section{Procedimiento}

Los encuestados fueron seleccionados mediante un muestreo no probabilístico por conveniencia a través de contactos realizados en el Instituto Católico Chileno de Migración (INCAMI) y el Centro Integrado de Atención al Migrante (CIAMI) de la Fundación Scalabrini, en Santiago de Chile. Los instrumentos fueron aplicados por los miembros del equipo de investigación, y de manera individual, en inmediaciones de dichas instituciones.

\section{Aspectos éticos}

El proyecto y los consentimientos informados fueron sometidos a la evaluación de un comité de ética científica acreditado. Se siguieron todos los procedimientos éticos necesarios para el respeto de los derechos humanos en el estudio con personas, tal como lo señalan los convenios internacionales y los comités de ética institucionales del país. Todos los encuestados participaron de manera voluntaria y fueron debidamente informados acerca del objetivo y las características de este estudio. Los participantes firmaron consentimientos informados en los cuales se aseguraba la confidencialidad y anonimato de la información producida, antes de contestar los instrumentos de evaluación. La información fue tratada de manera confidencial y se eliminó cualquier elemento que pudiera dar cuenta de la identidad de los sujetos.

\section{Análisis de datos}

Los datos fueron analizados por medio del software estadístico IBM SPPS, versión 24. En primer lugar, se realizaron los análisis de fiabilidad de los instrumentos, cuyos coeficientes alfa se presentaron en la sección precedente. Posteriormente, se realizó la prueba de KolmogorovSmirnov con la corrección Lilliefors para cada una de las variables, y se determinó la no normalidad de la distribución de los datos en la mayoría de ellas, por lo que se optó por utilizar las puntuaciones tipificadas (puntajes Z) para los análisis correlacionales de Pearson. Luego, se realizó el análisis de conglomerados con el fin de identificar grupos de sujetos basados en diferencias en sus puntajes en las orientaciones de aculturación; de este modo, los individuos cuyos puntajes exhibieron patrones similares a través de las variables fueron agrupados y diferenciados de otros grupos que mostraban patrones diferentes en sus puntajes (Mooi \& Sarstedt, 2011). Por último, para determinar la existencia de diferencias entre perfiles aculturativos en estrés de aculturación y bienestar psicológico, se utilizó la prueba $\mathrm{H}$ de Kruskal Wallis, además de las pruebas post hoc de Mann Whitney con la corrección de Bonferroni.

\section{Resultados}

Con el fin de determinar la posible influencia de aspectos sociodemográficos en las variables de estudio se realizaron análisis de comparación de medias según el sexo y el nivel educativo de los participantes, cuyos resultados arrojaron que no existen diferencias significativas entre hombres y mujeres ni según el nivel educativo en las orientaciones de aculturación, el estrés y el bienestar. Además, se realizó un 
análisis correlacional entre estas variables y el tiempo de permanencia en Chile, pero no se encontraron relaciones estadísticamente significativas entre ellas.

En la Tabla 1 se presentan los estadísticos descriptivos para las orientaciones de aculturación, el estrés por aculturación y el bienestar. En términos generales, la orientación a la integración presentó los niveles más altos de aceptación, seguida por la orientación a la separación y el individualismo; mientras que las orientaciones a la asimilación y, en particular, la marginación, fueron las menos respaldadas por la muestra.

En términos del estrés por aculturación, la preparación para emigrar representó la principal preocupación de los participantes, seguida por las preocupaciones derivadas del estatus jurídico y las preocupaciones económicas; mientras que los retos asociados a la adaptación sociocultural fueron reportados como los menos estresantes para los participantes, aunque en niveles cercanos al punto medio de la escala.

Finalmente, se reportaron niveles de bienestar psicológico por encima del punto de medio de la escala de uno a cinco puntos, en donde el propósito en la vida, el crecimiento personal y la autoaceptación presentaron los niveles más altos, seguidos por el dominio del entorno, la autonomía y las relaciones positivas con los otros.
Relaciones entre las orientaciones de aculturación, el estrés por aculturación y el bienestar psicológico

Para los análisis de correlaciones, y considerando los resultados obtenidos en las pruebas de comprobación de los supuestos de normalidad, se optó por trabajar con los valores tipificados (puntajes Z). Como se aprecia en la Tabla 2, la separación cultural se asoció positivamente con el estrés aculturativo y con todos sus componentes. Asimismo, la percepción de dificultades en la preparación de la emigración se vinculó con la preferencia por la mantención de las propias pautas culturales y con una actitud negativa hacia la incorporación de la cultura de la sociedad mayoritaria. Y la tendencia a la separación se relacionó de forma positiva tanto con el estrés experimentado debido al estatus jurídico de inmigrante como con el proceso de adaptación al nuevo entorno sociocultural.

Por otra parte, la orientación a la marginalización se relacionó con el estrés producido por aspectos objetivos del proceso migratorio, como lo son los problemas económicos y las dificultades relativas al estatus jurídico. Por otra parte, el individualismo aculturativo se asoció con un mayor estrés por los problemas económicos. No se encontraron relaciones entre la integración, la asimilación y el estrés, ni con sus dimensiones.

Tabla 1.

Estadísticos descriptivos $(N=194)$ para las orientaciones de aculturación, el estrés aculturativo y el bienestar psicológico

\begin{tabular}{|c|c|c|c|c|}
\hline & \multicolumn{4}{|c|}{ Kolmogorov-Smirnov $^{\mathrm{a}}$} \\
\hline & $\mathrm{Z}$ & $\mathrm{p}$ & $\mathrm{M}$ & $\mathrm{DE}$ \\
\hline \multicolumn{5}{|l|}{ Orientaciones de aculturación ${ }^{b}$} \\
\hline Separación & .150 & .000 & 4.39 & 1.86 \\
\hline Integración & .169 & .000 & 4.94 & 1.58 \\
\hline Asimilación & .401 & .000 & 1.71 & 1.31 \\
\hline Marginación & .474 & .000 & 1.48 & 1.21 \\
\hline Individualismo & .377 & .000 & 2.18 & 1.89 \\
\hline Estrés aculturativo $^{c}$ & .098 & .000 & 2.82 & 0.70 \\
\hline Adaptación Sociocultural & .073 & .014 & 2.57 & 1.06 \\
\hline Preocupaciones económicas & .073 & .013 & 2.87 & 0.83 \\
\hline Preparación para emigrar & .047 & $.200 *$ & 3.18 & 1.03 \\
\hline Estatus jurídico & .117 & .000 & 2.90 & 0.95 \\
\hline Bienestar psicológico $^{d}$ & .059 & $.200 *$ & 3.89 & 0.47 \\
\hline Autoaceptación & .149 & .000 & 4.10 & 0.61 \\
\hline Relaciones positivas & .082 & .003 & 3.60 & 0.73 \\
\hline Autonomía & .082 & .003 & 3.63 & 0.63 \\
\hline Dominio del entorno & .100 & .000 & 3.83 & 0.62 \\
\hline Crecimiento personal & .124 & .000 & 4.12 & 0.61 \\
\hline Propósito en la vida & .163 & .000 & 4.16 & 0.64 \\
\hline
\end{tabular}

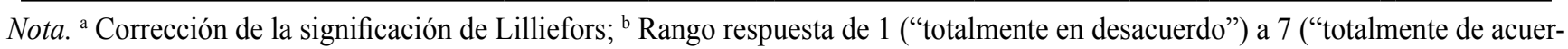
do"); c 1 ("nada estresante") a 5 ("muy estresante"); d 1 ("totalmente en desacuerdo") a 5 ("totalmente de acuerdo"). * Este es un límite inferior de la significación verdadera. 
Asimismo, con respecto a la relación entre las orientaciones de aculturación y el bienestar psicológico, la separación se vinculó con una percepción negativa de la capacidad para establecer vínculos sociales. El individualismo aculturativo se asoció negativamente con el bienestar psicológico, y, en específico, con la autoaceptación, las relaciones sociales, el dominio del entorno y el crecimiento personal. Por el contrario, la integración se relacionó de forma positiva con el crecimiento personal. Las orientaciones de asimilación y marginalización no se relacionaron con el bienestar psicológico percibido.

\section{Perfiles aculturativos}

Los resultados obtenidos mediante el análisis de conglomerados permitieron diferenciar tres grupos según sus preferencias aculturativas. Como se observa en la Tabla 3, el Grupo 1, que concentra al $53.1 \%$ de los participantes, presentó como característica central y distintiva una alta puntuación en la orientación a la integración cultural, acompañada por un puntaje medio en separación, y bajas puntuaciones en asimilación, marginalización e individualismo.
Esto indicaría que, si bien existe un fuerte interés por el biculturalismo, la mantención de la propia cultura sería condición para el intercambio cultural, lo que es reforzado por la baja tendencia tanto a la asimilación como hacia las estrategias que suponen el distanciamiento de ambas culturas. En consecuencia, este grupo ha sido denominado "integracionista".

El Grupo 2, que representa al $28.4 \%$ de la muestra, se distinguió por una alta separación o motivación por mantener su cultura de origen, aunque están dispuestos a adoptar algunos elementos de la sociedad receptora, tal como lo indica su puntaje medio-alto en integración. El individualismo, la marginalización y la asimilación surgen como alternativas menos deseables, lo que da cuenta de la relevancia de mantener la cultura de origen y el bajo interés por distanciarse de ella en favor de la incorporación de la cultura de recepción. Este grupo ha sido denominado "separatista".

Por último, el Grupo 3, en adelante "individualista moderado", en el que se encuentra el $18.5 \%$ de los encuestados, mostró una alta tendencia al individualismo, en la que se

Tabla 2.

Matriz de correlaciones de Pearson entre el estrés aculturativo, el bienestar psicológico y las orientaciones de aculturación

\begin{tabular}{lccccc}
\hline & Separación & Integración & Asimilación & Marginalización & Individualismo \\
\hline Estrés aculturativo & $.33^{* *}$ & -.13 & -.10 & .09 & .06 \\
Adaptación Sociocultural & $.27^{* * *}$ & -.10 & -.11 & .01 & -.05 \\
Preocupaciones económicas & .13 & -.11 & .05 & $.15^{*}$ & $.17^{*}$ \\
Preparación para emigrar & $.20^{* *}$ & -.03 & -.13 & .06 & .02 \\
Estatus jurídico & $.24 * *$ & -.12 & -.07 & $.18^{*}$ & .10 \\
Bienestar psicológico & -.12 & .13 & -.03 & -.07 & $-.16^{*}$ \\
Autoaceptación & -.05 & .12 & .05 & -.02 & $-.14^{*}$ \\
Relaciones positivas & $-.15^{*}$ & .09 & -.09 & -.12 & $-.18^{*}$ \\
Autonomía & -.07 & .04 & .08 & -.13 \\
Dominio del entorno & .01 & .10 & .05 & -.05 & $-.15^{*}$ \\
Crecimiento personal & -.09 & .04 & .10 & -.01 & $-.16^{*}$ \\
Propósito en la vida & -.05 & & & -.11 \\
\hline
\end{tabular}

Nota. $* \mathrm{p}<.05 . * * \mathrm{p}<.01 . * * * \mathrm{p}<.001$. Para las magnitudes de las correlaciones se sugiere optar por una interpretación de pequeña asociación $(\mathrm{r}>.10)$, mediana asociación $(\mathrm{r}>.30)$ y gran asociación $(\mathrm{r}>.50)^{1}$.

\footnotetext{
${ }^{1}$ En los criterios del tamaño del efecto sugeridos por Cohen en 1962 se plantea que una correlación de $r=.20$ es pequeña, que una de $r=.40$ es mediana, y que una de de $\mathrm{r}=.60$ es grande. Sin embargo, el mismo autor modificó su apreciación en 1988, y señaló que las correlaciones de $.10, .30$ y .50 debían ser pequeñas, medianas y grandes, respectivamente. Incluso, en 1992 Cohen reconoció que las definiciones que él mismo realiza son subjetivas. Debido a lo anterior es que el mismo autor (Cohen, Cohen, Wets \& Aiken, 2003) planteó que hay que seguir tres estrategias específicas (y en estricto orden) para la interpretación del tamaño de las asociaciones: (a) ¿qué otras relaciones en estudios anteriores han encontrado otros investigadores que me planteen un rango del tamaño del efecto?; (b) en algunas áreas de investigación, un investigador puede plantear un tamaño mínimo del efecto en la población, el cual tendría una importancia práctica o teórica; y (c) como tercera estrategia, y solo cuando las anteriores no son suficientes, utilizar la convención sugerida por el mismo Cohen (.10, .30 y .50) (Cohen et al., 2003, p. 52).
} 
Tabla 3.

Estadísticos descriptivos para las orientaciones de aculturación por conglomerados

\begin{tabular}{|c|c|c|c|c|}
\hline & Orientaciones de aculturación ${ }^{\mathrm{a}}$ & $N$ & $M$ & $D E$ \\
\hline \multirow{5}{*}{ Integracionista } & Separación & 103 & 3.44 & 1.35 \\
\hline & Integración & 103 & 5.26 & 1.31 \\
\hline & Asimilación & 103 & 1.81 & 1.28 \\
\hline & Marginación & 103 & 1.42 & 1.09 \\
\hline & Individualismo & 103 & 1.30 & 0.65 \\
\hline \multirow{5}{*}{ Separatista } & Separación & 55 & 6.53 & 0.74 \\
\hline & Integración & 55 & 4.33 & 1.93 \\
\hline & Asimilación & 55 & 1.09 & 0.29 \\
\hline & Marginación & 55 & 1.35 & 1.06 \\
\hline & Individualismo & 55 & 1.78 & 1.66 \\
\hline \multirow{5}{*}{ Individualista moderado } & Separación & 36 & 3.83 & 1.63 \\
\hline & Integración & 36 & 4.97 & 1.46 \\
\hline & Asimilación & 36 & 2.36 & 1.87 \\
\hline & Marginación & 36 & 1.86 & 1.62 \\
\hline & Individualismo & 36 & 5.28 & 1.36 \\
\hline
\end{tabular}

Nota. a Rango respuesta de 1 ("totalmente en desacuerdo") a 7 ("totalmente de acuerdo").

atiende en mayor medida a la dimensión interpersonal y se le otorga un menor valor a la pertenencia grupal. La presencia de un puntaje medio-alto en integración reflejaría su disposición al intercambio cultural, y la tendencia moderada hacia la separación cultural indicaría que en el plano intergrupal existiría el deseo de mantener su cultura de origen, lo que se relacionaría con sus bajas puntuaciones en asimilación y marginalización.

Estrés de aculturación y bienestar psicológico según los perfiles aculturativos

Debido a la no normalidad de los datos, se utilizó el test de Kruskal Wallis para estudiar la existencia de diferencias significativas entre el estrés y el bienestar psicológico, según los perfiles aculturativos. Tal como se observa en la Tabla 4, los resultados muestran que existen diferencias entre los grupos en cuanto al estrés de aculturación global $\left(\mathrm{H}_{(2)}=20.57, \mathrm{p}<.01\right)$.

Específicamente, las pruebas post hoc de Mann Whitney, con la corrección de Bonferroni, indican que el grupo Integracionista presentó un rango medio significativamente menor que el grupo Separatista, $\mathrm{U}\left(\mathrm{N}_{\text {Integracionistas }}=103\right.$, $\left.\mathrm{N}_{\text {Separatistas }}=55\right)=1623.00, \mathrm{z}=-4.42, \mathrm{p}<.001$, sin embargo, el grupo individualista moderado no difirió significativamente de los demás perfiles.

En la dimensión de adaptación sociocultural también se evidenciaron diferencias significativas entre los perfiles aculturativos $\left(\mathrm{H}_{(2)}=11.61, \mathrm{p}<.01\right)$, donde el grupo integracionista mostró un rango medio significativamente menor que el grupo Separatista $\left(\mathrm{U}\left(\mathrm{N}_{\text {Integracionistas }}=103\right.\right.$, $\left.\left.\mathrm{N}_{\text {Separatistas }}=55\right)=1888.000, \mathrm{z}=-3.45, \mathrm{p}<.001\right)$, mientras que el grupo individualista moderado no presentó diferencias significativas con respecto a los otros perfiles.

En cuanto a la dimensión de preparación para la emigración, se observaron diferencias significativas entre los grupos $\left(\mathrm{H}_{(2)}=7.70, \mathrm{p}<.05\right)$, ya que el grupo Integracionista presentó un rango medio significativamente menor que el grupo separatista $\left(\mathrm{U}\left(\mathrm{N}_{\text {Integracionistas }}=103, \mathrm{~N}_{\text {Separatistas }}=55\right)=2099.50\right.$, $\mathrm{z}=-2.68, \mathrm{p}<.01)$. El grupo individualista moderado no mostró diferencias significativas con respecto al resto de los perfiles.

Asimismo, en la dimensión de estrés por el estatus jurí$\operatorname{dico}\left(\mathrm{H}_{(2)}=13.53, \mathrm{p}<.001\right)$ las pruebas post hoc muestran que este patrón se replica, ya que el grupo integracionista mostró una diferencia significativa con el grupo separatista $\left(\mathrm{U}\left(\mathrm{N}_{\text {Integracionistas }}=103, \mathrm{~N}_{\text {Separatistas }}=55\right)=1833.50, \mathrm{z}=-3.66\right.$, $\mathrm{p}<.001$ ), y el individualista moderado no se diferenció significativamente de los demás perfiles. No se encontraron diferencias significativas entre los grupos en la dimensión preocupaciones económicas. Los tamaños del efecto para las diferencias entre los grupos se muestran en la Tabla $6^{2}$.

Por otra parte, en la Tabla 5 se observa que existen diferencias significativas en el bienestar psicológico global a

2 Los autores de este trabajo plantean que estos puntos de corte para los tamaños del efecto son convenciones determinadas por investigadores específicos, por lo que son arbitrarios. Dado lo anterior, hay que interpretarlos como continuos y referenciales, pero no como regla absoluta. 
Tabla 4.

Test de Kruskal-Wallis y rangos medios de los perfiles aculturativos para el estrés de aculturación y sus dimensiones

\begin{tabular}{|c|c|c|c|c|}
\hline & & $\mathrm{N}$ & Rango medio & $X^{2(g l)}$ \\
\hline \multirow{3}{*}{ Estrés aculturativo global } & Integracionista & 103 & 81.89 & $20.57(2)^{* * a}$ \\
\hline & Separatista & 55 & 124.13 & \\
\hline & Individualista moderado & 36 & 101.49 & \\
\hline \multirow{3}{*}{ Adaptación sociocultural } & Integracionista & 103 & 87.27 & $11.61(2)^{* * a}$ \\
\hline & Separatista & 55 & 118.95 & \\
\hline & Individualista moderado & 36 & 94.00 & \\
\hline \multirow{3}{*}{ Preocupaciones económicas } & Integracionista & 103 & 90.24 & $3.70(2)$ \\
\hline & Separatista & 55 & 105.07 & \\
\hline & Individualista moderado & 36 & 106.71 & \\
\hline \multirow{3}{*}{ Preparación para emigrar } & Integracionista & 103 & 89.61 & $7.69(2)^{* a}$ \\
\hline & Separatista & 55 & 115.14 & \\
\hline & Individualista moderado & 36 & 93.14 & \\
\hline \multirow{3}{*}{ Estatus jurídico } & Integracionista & 103 & 84.85 & $13.53(2)^{* * a}$ \\
\hline & Separatista & 55 & 119.09 & \\
\hline & Individualista moderado & 36 & 100.69 & \\
\hline
\end{tabular}

Nota. ${ }^{*} \mathrm{p}<.05,{ }^{* *} \mathrm{p}<.01$. Pruebas post hoc de Mann-Withney con corrección de Bonferroni. ${ }^{a}$ Diferencias significativas al $\mathrm{p}<.01$ entre integracionistas vs. separatistas.

Tabla 5.

Test de Kruskal-Wallis y rangos medios de los perfiles aculturativos para el bienestar psicológico y sus dimensiones

\begin{tabular}{|c|c|c|c|c|}
\hline & & $\mathrm{N}$ & Rango medio & $X^{2(g l)}$ \\
\hline \multirow{3}{*}{ Bienestar psicológico global } & Integracionista & 103 & 108.63 & $8.63(2)^{* a}$ \\
\hline & Separatista & 55 & 85.20 & \\
\hline & Individualista moderado & 36 & 84.46 & \\
\hline \multirow{3}{*}{ Autoaceptación } & Integracionista & 103 & 106.16 & $6.36(2) * b$ \\
\hline & Separatista & 55 & 92.44 & \\
\hline & Individualista moderado & 36 & 80.46 & \\
\hline \multirow{3}{*}{ Relación positiva } & Integracionista & 103 & 106.50 & $5.72(2)$ \\
\hline & Separatista & 55 & 88.39 & \\
\hline & Individualista moderado & 36 & 85.68 & \\
\hline \multirow{3}{*}{ Autonomía } & Integracionista & 103 & 104.41 & $3.94(2)$ \\
\hline & Separatista & 55 & 93.31 & \\
\hline & Individualista moderado & 36 & 84.13 & \\
\hline \multirow{3}{*}{ Dominio del entorno } & Integracionista & 103 & 104.63 & $3.64(2)$ \\
\hline & Separatista & 55 & 90.61 & \\
\hline & Individualista moderado & 36 & 87.63 & \\
\hline \multirow{3}{*}{ Crecimiento personal } & Integracionista & 103 & 110.99 & $12.96(2)^{* * a \mathrm{~b}}$ \\
\hline & Separatista & 55 & 82.85 & \\
\hline & Individualista moderado & 36 & 81.28 & \\
\hline \multirow{3}{*}{ Propósito en la vida } & Integracionista & 103 & 106.17 & $5.35(2)$ \\
\hline & Separatista & 55 & 87.35 & \\
\hline & Individualista moderado & 36 & 88.22 & \\
\hline
\end{tabular}

Nota: ${ }^{*} \mathrm{p}<.05,{ }^{* *} \mathrm{p}<.01$. Pruebas post hoc de Mann Wihitney Bonferroni. ${ }^{\text {a }}$ Diferencias significativas al $\mathrm{p}<.05$ entre integracionistas vs. separatistas. ${ }^{b}$ Diferencias de medias significativas al $\mathrm{p}<.05$ de integracionistas vs. individualistas moderados. 
partir del perfil de aculturación $\left(\mathrm{H}_{(2)}=8.63, \mathrm{p}<.05\right)$. Así, de acuerdo con las comparaciones post hoc, se encontró que el grupo integracionista presentó un rango medio significativamente mayor que el grupo individualista moderado, $\mathrm{U}\left(\mathrm{N}_{\text {Integracionistas }}=103, \mathrm{~N}_{\text {Individualistas moderados }}=36\right)=2156.50$, $\mathrm{z}=-2.47, \mathrm{p}<.001$. El grupo separatista no presentó diferencias significativas con respecto a los otros grupos.

También, se presentaron diferencias significativas en la dimensión autoaceptación $\left(\mathrm{H}_{(2)}=6.36, \mathrm{p}<.05\right)$, ya que el grupo integracionista presentó un rango medio significativamente mayor que el grupo individualista moderado $\left(\mathrm{U}\left(\mathrm{N}_{\text {Integracionistas }}=103, \mathrm{~N}_{\text {Individualistas moderados }}=36\right)=1354.50\right.$, $\mathrm{z}=-2.43, \mathrm{p}<.001$ ), aunque el grupo separatista no se diferenció de manera significativa de ninguno de los anteriores. Además, se observaron diferencias en la dimensión de crecimiento personal $\left(\mathrm{H}_{(2)}=12.96, \mathrm{p}<.01\right)$, donde el grupo integracionista mostró un rango medio significativamente mayor que el grupo separatista $\left(\mathrm{U}\left(\mathrm{N}_{\text {Integracionistas }}=103\right.\right.$, $\left.\left.\mathrm{N}_{\text {Individualistas moderados }}=36\right)=1996.50, \mathrm{z}=-3.09, \mathrm{p}<.01\right)$, y que el individualista moderado $\left(\mathrm{U}\left(\mathrm{N}_{\text {Integracionistas }}=103\right.\right.$, $\left.\mathrm{N}_{\text {Individualistas moderados }}=36\right)=1300.50, \mathrm{z}=-2.69, \mathrm{p}<.01$ ). No se encontraron diferencias significativas entre en los grupos en su percepción de dominio del entorno, relaciones positivas, autonomía y propósito en la vida. Los tamaños del efecto para las diferencias entre perfiles aculturativos se presentan en la Tabla 6 .

\section{Discusión}

Los resultados obtenidos muestran que, al igual que en múltiples estudios previos en diferentes contextos culturales (Sam \& Berry, 2010), la integración se presentó como la orientación preferida de los participantes, seguida por la separación; además de que la asimilación y, sobre todo, la marginalización, se presentaron como las orientaciones menos respaldadas, lo cual evidencia la importancia que tienen el mantenimiento de la identidad cultural para las personas migrantes de este trabajo (Sam \& Berry, 2010).

Ahora bien, uno de los resultados más llamativos del presente estudio refiere a la emergente preferencia por las estrategias aculturativas individualistas, escasamente documentadas en investigaciones antecedentes en nuestro contexto.

Una de las primeras referencias sobre la importancia de considerar las tendencias individualistas dentro del marco de análisis de la aculturación de poblaciones migrantes proviene del Modelo Interactivo, de Bourhis et al. (1997). En este, los autores reconocen que, en algunos casos, bajos niveles de mantenimiento cultural, acompañados de un distanciamiento frente a la cultura receptora, no necesariamente implican marginalización. Este distanciamiento puede deberse a que los migrantes se ven a sí mismos más como individuos y menos como miembros de un colectivo, y su orientación hacia la sociedad receptora puede estar motivada por estrategias

Tabla 6.

Tamaños del efecto (d de Cohen) para las diferencias de medias entre los perfiles aculturativos en el estrés de aculturación y el bienestar psicológico

\begin{tabular}{|c|c|c|c|}
\hline Variable & $\begin{array}{c}\text { Integracionista- } \\
\text { Separatista }\end{array}$ & $\begin{array}{c}\text { Integracionista- } \\
\text { Individualista mod- } \\
\text { erado }\end{array}$ & $\begin{array}{c}\text { Separatista- } \\
\text { Individualista mod- } \\
\text { erado }\end{array}$ \\
\hline Estrés aculturativo global & -.75 & n. s. & n. s. \\
\hline Adaptación sociocultural & -.59 & n. s. & n. s. \\
\hline Preocupaciones económicas & n. s. & n. s. & n. s. \\
\hline Preparación para emigrar & -.48 & n. s. & n. s. \\
\hline Estatus jurídico & -.65 & n. s. & n. s. \\
\hline Bienestar psicológico global & .41 & n. s. & n. s. \\
\hline Autoaceptación & n. s. & .52 & n. s. \\
\hline Relación positiva & n. s. & n. s. & n. s. \\
\hline Autonomía & n. s. & n. s. & n. s. \\
\hline Dominio del entorno & n. s. & n. s. & n. s. \\
\hline Crecimiento personal & .32 & .55 & n. s. \\
\hline Propósito en la vida & n. s. & n. s. & n. s. \\
\hline
\end{tabular}

Nota. Puntos de corte para el tamaño de efecto de la d de Cohen: $.20=$ pequeño; $.50=$ mediano; y $.80=$ grande $($ Cohen, 1988; Ellis, 2010). 
de movilidad individual (Tajfel \& Turner, 1979). Puede que este tipo de tendencias resulten más probables en migrantes provenientes de sociedades individualistas en donde los valores del logro y la realización individual son altamente promovidos culturalmente, en contraposición a aquellos migrantes provenientes de culturas colectivistas en donde las personas tienden a subordinar sus objetivos personales a los objetivos de los grupos a los que pertenecen (Triandis, Bontempo, Villareal, Asai \& Lucca, 1988). Sin embargo, los resultados obtenidos revelan que dichas tendencias no son exclusivas de migrantes de sociedades típicamente individualistas, sino que se observan también en las sociedades latinoamericanas, caracterizadas en la literatura como contextos típicamente colectivistas o interdependientes (Triandis et al., 1988).

Estas tendencias pueden explicarse, por una parte, porque pueden ser reflejo de las características particulares de la muestra estudiada, ya que los grupos migrantes pueden clasificarse en dos grandes categorías: (a) quienes hacen migraciones "voluntarias", es decir, aquellos que abandonan sus países de origen de manera relativamente libre, en busca de superación personal a través del empleo, oportunidades económicas y de estudio, por matrimonio o para unirse a familiares que han inmigrado anteriormente; $y$ (b) quienes realizan migraciones involuntarias, como en el caso de las personas refugiadas y solicitantes de asilo, que son desplazadas forzosamente por la guerra, la persecución o los desastres (Berry, 2005; Bourhis, et al., 1997).

En particular, los participantes de este estudio se ubican dentro de la primera categoría, por lo que su proyecto de migrar parece implicar una serie de motivaciones, metas y características de personalidad potencialmente vinculadas al individualismo. Esto concuerda con que, en otras latitudes, la investigación ha mostrado que las personas migrantes voluntarias presentan mayores niveles de agencia personal y locus de control interno que sus compatriotas no migrantes (Ayçiçegi-Dinn \& Caldwell-Harris, 2011), y que en general los valores de individualismo y compromiso igualitario se encuentran positivamente relacionados con la movilidad y la disposición de las poblaciones para migrar a otras ciudades y países (Basabe \& Ros, 2005).

Por otra parte, estas tendencias podrían ser un reflejo de posibles cambios en los valores culturales, reflejo de la globalización y movilización intrarregional acelerada (Martínez \& Vono, 2005). Al respecto, varios estudios indican que los aspectos clásicos de la ética protestaste del trabajo atribuidos a las sociedades capitalistas individualistas forman parte también de los valores de las sociedades tradicionalmente clasificadas como colectivistas, lo cual es congruente con la presencia de actitudes competitivas en sociedades colectivistas (Lynn \& Martin, 1995). Estos cambios han llevado a reconsiderar las nociones tradicionales de colectivismo e individualismo, así como la clasificación dicotómica de las sociedades en estas dos categorías (Oyserman, Coon $\&$ Kemmelmeier, 2002).

Estas hipótesis no pueden ser abordadas apropiadamente con los datos del presente estudio, pero proporcionan evidencia importante para considerar en futuras investigaciones, en las cuales la comparación de diferentes tipos de poblaciones migrantes y la medición de valores resultarían de gran relevancia.

En términos del estrés por aculturación, los resultados coinciden con antecedentes en Latinoamérica (UgaldeWatson et al., 2011), pues evidencian que los preparativos para la salida y la emigración representan la principal fuente de estrés percibido, seguida por las preocupaciones derivadas del estatus migratorio y las preocupaciones económicas. $\mathrm{Si}$ bien la emigración trae consigo oportunidades de mejora en la calidad de vida de muchas personas, esta se ve acompañada también por procesos complejos de desvinculación cultural y familiar que representan importantes fuentes de estrés, las cuales, a su vez, pueden afectar los procesos de adaptación sociocultural y psicosocial (Bobowik et al., 2014). Igualmente, los hallazgos de este trabajo sugieren que las barreras estructurales que impiden la regularización de la situación jurídica, el empleo, la vivienda y la salud de la población migrante se perfilan como importantes fuentes de tensión y estrés que requieren de mayor estudio.

En concordancia con los resultados de investigaciones previas (Basabe, Zlobina \& Páez, 2004; Elgorriaga, Martínez-deTaboada \& Arnoso-Martínez, 2014; Smith \& Bond, 1993; Ward, Bochner \& Fumham et al., 2001), los niveles de estrés reportados ante los retos que conlleva la adaptación a las costumbres, prácticas y valores de la sociedad receptora son bastante menores, probablemente por la presencia de una matriz cultural común, pero apuntan al potencial impacto negativo de las actitudes de la sociedad receptora sobre los procesos de adaptación psicológica, tal como se ha evidenciado en otras investigaciones (Cárdenas, 2006; González et al., 2010; Stefoni, 2011; Valenzuela et al., 2018; Sirlopú \& van Oudenhoven, 2013).

En términos de los vínculos entre las orientaciones de aculturación y el estrés aculturativo, los resultados corroboraron la asociación positiva de la separación cultural con el estrés aculturativo, con un vínculo específico con el proceso de preparación de la emigración, la adaptación sociocultural a la sociedad receptora y el estrés producido por el estatus jurídico de inmigrante. Este hallazgo es consistente con el de estudios anteriores (Berry \& Sabatier, 2010; Basabe et al, 2009; Elgorriaga, 2011; Navas et al., 2004; Urzúa et al., 2016) que indican que la preferencia por la mantención de la cultura de origen y el rechazo del aprendizaje de la cultura de recepción se vinculan con diversas dificultades 
en el ajuste al nuevo contexto. Además, la orientación a la marginalización se relacionó positivamente con el estrés producido por el aspecto económico y el estatus jurídico de inmigrante, en línea con otras investigaciones que vinculan esta actitud con el riesgo de exclusión social (Berry \& Sabatier, 2010; Basabe et al, 2009; Elgorriaga, 2011; Navas et al., 2004; Yoon et al, 2013).

Adicionalmente, en este estudio el individualismo aculturativo se asoció positivamente con el estrés por problemas económicos. Es posible que el interés por la movilidad individual esté vinculado con la preocupación por el logro de recursos en el proceso migratorio, lo que produce un elevado malestar. Al respecto, estudios metaanalíticos precedentes han mostrado que en poblaciones inmigrantes los valores de promoción personal a través del logro se asocian a índices de malestar subjetivo (Bobowik, Basabe, Páez \& Jimenez-Aristizabal, 2010; Bobowik, Basabe, Páez, Jimenez \& Bilbao, 2011), evidencia que parece coincidir con la idea de que el estrés aculturativo reportado en este estudio es reflejo de los esfuerzos por la movilidad social ascendente. Asimismo, es posible que el establecimiento de redes con otros inmigrantes y con la sociedad receptora sea obstaculizado por la tendencia individualismo, lo que puede limitar el acceso al apoyo en situaciones de dificultad.

Por otra parte, a diferencia de otras investigaciones que destacan la integración como la orientación que facilita un mejor afrontamiento del proceso de adaptación al país receptor (Basabe et al, 2009; Chen et al., 2008; Kuo, 2014; Torres et al., 2012; Urzúa et al., 2017; Yáñez \& Cárdenas, 2010), los resultados obtenidos no reportaron relaciones entre dicha actitud y el estrés aculturativo, y tampoco con la asimilación. En conjunto, estos resultados podrían indicar que, en este estudio, el rechazo y la ausencia de interés por la cultura local se asocian con la capacidad para afrontar la falta de recursos materiales y los problemas derivados de la situación jurídica, más que la adopción de la cultura de acogida (integración, asimilación).

Con respecto a la relación entre las orientaciones de aculturación y el bienestar psicológico, la separación se relacionó negativamente con el establecimiento de vínculos sociales, lo que refleja la dificultad para relacionarse con la población local. En cambio, la orientación a la integración se relacionó positivamente con la dimensión crecimiento personal del bienestar, en línea con resultados de las investigaciones de Silva et al. (2016) y Yáñez y Cárdenas (2010). A diferencia de los resultados obtenidos por estos dos estudios, las orientaciones de asimilación y marginalización encontradas en este trabajo no se relacionaron con el bienestar psicológico percibido.

Específicamente, y en concordancia con lo propuesto por algunos autores (van Leeuwen et al, 2010; van Leeuwen et al., 2014), el individualismo aculturativo se asoció de forma negativa con el bienestar en general y con las dimensiones de autoaceptación, relaciones sociales, dominio del entorno $\mathrm{y}$ crecimiento personal. Esto podría sugerir que la búsqueda de movilidad social como estrategia para satisfacer las propias necesidades y alcanzar metas individuales tiene como correlato, paradójicamente, un empobrecimiento en la capacidad para desarrollar potencialidades personales, pues implica el distanciamiento del soporte y los aprendizajes que pueden proporcionar las culturas de origen y recepción.

Por otra parte, cabe destacar que en este estudio la marginalización, que ha sido vinculada con menor bienestar por estudios anteriores (Yoon et al., 2013), no presentó una relación significativa con esta variable, y, en cambio, el individualismo se asoció negativamente al bienestar. Aun cuando el elemento común a estas dos orientaciones es el bajo interés por el encuentro cultural con el otro, es importante señalar que las condiciones del contexto chileno promueven la creencia y búsqueda de la movilidad social individual como vía de acceso a recursos (Mera et al., 2017), lo que puede facilitar la emergencia de actitudes individualistas.

Ahora bien, asumiendo que las orientaciones de aculturación en la vida cotidiana operan de manera dinámica e interrelacionada (Barrette et al., 2004), los resultados por el agrupamiento de sujetos según sus estilos —o tipologías-de orientaciones aculturativas ayudan a matizar las relaciones establecidas mediante los análisis correlacionales entre las variables. Así, se observa que el predominio de la integración es más salutogénico que la tendencia a la separación cultural como orientación preeminente, lo que facilita un mejor afrontamiento de los estresores del proceso migratorio. Esto confirma los hallazgos informados por estudios anteriores con respecto al proceso de adaptación al nuevo contexto (Basabe et al, 2009; Chen et al., 2008; Kuo, 2014; Torres et al., 2012; Urzúa et al., 2017; Yáñez \& Cárdenas, 2010).

Finalmente, los resultados señalan que cuando la integración es más saliente, el bienestar es mayor que en presencia del individualismo como orientación predominante, resultado consistente con otras investigaciones que han relacionado el biculturalismo con el bienestar psicológico desde el enfoque de la eudaemonia (Silva et al, 2016; Yáñez $\&$ Cárdenas, 2010). Esto último aporta evidencia con respecto al individualismo como orientación de aculturación escasamente considerada en la literatura latinoamericana, especialmente en relación con la salud psicológica (van Leeuwen et al., 2010; van Leeuwen et al., 2014), y cuya posible influencia negativa puede relacionarse con la relevancia de la pertenencia grupal como vía para acceder a recursos sociales, afectivos y materiales que facilitan la adaptación en procesos de transición psicosocial y cultural, 
en especial en contextos donde existen barreras para la inclusión (Mera et al., 2014). Es necesario resaltar que, en este estudio, los perfiles aculturativos obtenidos no recogen la tipología de la marginalización que tradicionalmente ha sido vinculada con menor bienestar (Yoon et al., 2013). Esto podría deberse al hecho de que los participantes de este estudio fueron contactados a través de instituciones de ayuda al migrante, es decir, se trata de personas que presentan algún grado de vinculación social, por lo que es poco probable que en estos contextos se pueda encontrar sujetos con predominio de dicha orientación.

Cabe señalar, como principales limitaciones de este estudio, la utilización de una muestra de conveniencia en el marco de un diseño transversal, que no permite observar la relación entre las orientaciones de aculturación, el estrés y el bienestar a lo largo del tiempo. Además, el tamaño de la muestra y la diversidad de nacionalidades, que no permiten diferenciar los grupos de inmigrantes según su país de origen, y que tampoco se consideró el grado en que los participantes perciben que su migración fue voluntaria. Asimismo, que no se utilizaron medidas de conductas de aculturación que permitieran una aproximación más compleja al fenómeno estudiado. Igualmente, que algunos de los instrumentos presentaron coeficientes de consistencia interna relativamente bajos que pudieron haber afectado la posibilidad para detectar las relaciones entre las variables.

A pesar de dichas limitaciones, los resultados presentados contribuyen a la comprensión de la incidencia de la aculturación en el bienestar, donde se resaltan, principalmente, los beneficios del biculturalismo, y se alerta sobre las posibles consecuencias psicológicas del individualismo, aspecto que surge como amenaza para el bienestar psicológico, y que, a pesar de esto, ha sido menos estudiado. Se considera que esta información aporta a una línea de investigación que, si bien en el contexto latinoamericano es todavía incipiente, resulta de gran relevancia como insumo para el diseño y la implementación de acciones y políticas públicas en materia de inmigración.

\section{Referencias}

Achotegui, J. (2006). Estrés límite y salud mental: el síndrome del inmigrante con estrés crónico y múltiple (Síndrome de Ulises). Migraciones, 19, 59-85. doi: 10.1016/ S0304-4858(09)74665-7

Ayçiçegi-Dinn, A., \& Caldwell-Harris, C. L. (2011). Individualism-collectivism among Americans, Turks and Turkish immigrants to the US. International Journal of Intercultural Relations, 35(1), 9-16. doi: 10.1016/j. ijintrel.2010.11.006
Azurmendi, M. J., \& Larrañaga, N. (2008). La inmigración emergente en la Comunidad Autónoma Vasca (CAV) desde la sociedad de acogida: la aculturación. En J. F. Morales, C. Huici, A. Gómez y E. Gaviria (eds.), Método, teoría e investigación en psicología social (pp. 487-512). Madrid: Pearson-Prentice Hall.

Barrette, G., Bourhis, R. Y., Personnaz, M., \& Personnaz, B. (2004). Acculturation orientations of French and North African undergraduates in Paris. International Journal of Intercultural Relations, 28(5), 415-438. doi: 10.1016/j. ijintrel.2004.08.003

Basabe, N., Páez, D., Aierdi, X., \& Jiménez-Aristizabal, A. (2009). Calidad de vida, bienestar subjetivo y salud: inmigrantes en la CAPV. Zumaia: Ikuspegi, Observatorio Vasco de Inmigración.

Basabe, N., \& Ros, M. (2005). Cultural dimensions and social behavior correlates: Individualism-Collectivism and Power Distance. International Review of Social Psychology, 18(1), 189-225. Recuperado de https:// www.ehu.eus/documents/1463215/1504238/./ d8caafd9-c2ef-41cc-b809-f905f4f190c9

Basabe, N., Zlobina, A., \& Páez, D. (2004). Integración sociocultural y adaptación psicológica de los inmigrantes extranjeros en el País Vasco. Cuadernos Sociológicos Vascos, 15, 1-136. Recuperado de http://www.euskadi.eus/contenidos/ documentacion/cuaderno_sociologico_vasco_15/es_cu_ soc15/adjuntos/csv15.pdf

Berry, J. W. (2005). Acculturation: Living successfully in two cultures. International Journal of Intercultural Relations, 29(6), 697-712. doi: 10.1016/j.ijintrel.2005.07.013

Berry, J., \& Sabatier, C. (2010). Acculturation, discrimination, and adaptation among second generation immigrant youth in Montreal and Paris. International Journal of Intercultural Relations, 34(3), 191-207. doi: 10.1016/j. ijintrel.2009.11.007

Bobowik, M., Basabe, N., \& Páez, D. (2014). The bright side of migration: Hedonic, psychological, and social well-being in immigrants in Spain. Social Science Research, 51, 189-204. doi: 10.1016/j.ssresearch.2014.09.011

Bobowik, M., Basabe, N., Páez, D., Jiménez, A., \& Bilbao, Á. (2011). Personal values and well-being among Europeans, Spanish natives and immigrants to Spain: Does the culture matter? Journal of Happiness Studies, 12(3), 401-419. doi: 10.1007/s10902-010-9202-1

Bourhis R. Y., \& Barrette G. (2006). Notes on the Immigrant Acculturation Scale (IAS) (Working Paper, LECRI). Montreal, Quebec, Canada: Département de psychologie, Université du Québec à Montréal, Canada.

Bourhis, R. Y., Moïse, L. C., Perreault, S., \& Senécal, S. (1997). Towards an Interactive Acculturation Model: A Social Psychological Approach. International Journal of Psychology, 32(6) 369-386. doi: 10.1080/002075997400629

Cárdenas, M. (2006). Y verás cómo quieren en Chile: Un estudio sobre el prejuicio hacia los inmigrantes bolivianos por 
parte de jóvenes chilenos. Última Década, 24, 103-129. doi: 10.4067/S0718-22362006000100006

Chen, S., Benet-Martínez, V., \& Bond, M, H. (2008). Bicultural identity, bilingualism, and psychological adjustment in multicultural societies: immigration-based and globalizationbased acculturation. Journal of Personality, 76(4), 803-838. doi: 10.1111/j.1467-6494.2008.00505.x

Cohen, J. (1988). Statistical Power Analysis for the Behavioral Sciences. New York: Lawrence Erlbaum Associates.

Cohen, J., Cohen, P., West, S. G., \& Aiken, L. S. (2003). Applied multiple regression/correlation analysis for the behavioral sciences (3. ${ }^{\text {a }}$ ed.). Mahwah, N. J., EE. UU.: Lawrence Erlbaum Associates Publishers.

Díaz, D., Rodríguez-Carvajal, R., Blanco, A., Moreno-Jiménez, B., Gallardo, I., Valle, C., \& Van Dierendonck, D. (2006). Adaptación española de las escalas de bienestar psicológico de Ryff. Psicothema, 18(3), 572-577. Recuperado de http:// www.psicothema.com/psicothema.asp?id=3255

Elgorriaga, E. (2011). Ajuste Psicológico y Salud Mental de la población inmigrante: Influencia del Género y la Cultura (tesis doctoral). Universidad del País Vasco, Facultad de Psicología, España. Recuperado de https://addi.ehu.es/ bitstream/10810/12204/1/elgorriaga\%20astondoa.pdf

Elgorriaga, E., Martínez, C., \& Arnoso, A. (2014). La importancia del género y el origen cultural en los procesos migratorios y en la satisfacción con la vida de la población inmigrante. Boletín de Psicología, 112, 7-34. Recuperado de https://www.uv.es/seoane/boletin/previos/N112-1.pdf

Ellis, P. (2010). The essential guide to effect sizes: statistical power, meta-analysis, and the interpretation of research results. Cambridge, Reino Unido: Cambridge University Press.

Galaz, C., Poblete, R., \& Frías, C. (2017). Las operaciones de exclusión de personas inmigradas a través de las políticas públicas en Chile. Revista del CLAD Reforma y Democracia, 68, 169-204. Recuperado de http://old.clad. org/portal/publicaciones-del-clad/revista-clad-reforma-de mocracia/articulos/068-junio-2017/las-operaciones-de-ex clusion-de-personas-inmigradas-a-traves-de-las-politicaspublicas-en-chile

González, R., Sirlopú, D., \& Kessler, T. (2010). Prejudice among Peruvians and Chileans as a Function of Identity, Intergroup Contact, Acculturation Preferences, and Intergroup Emotions. Journal of Social Issues, 66(4), 803824. doi: 10.1111/j.1540-4560.2010.01676.x

Hernández, R., Baptista, P., \& Fernández, C. (2010). Metodología de la investigación. Ciudad de México, México: McGraw-Hill.

Instituto Nacional de Estadísticas. (2018). Resultados Censo 2017. Recuperado de https://redatam-ine.ine.cl/redbin/ RpWebEngine.exe/Portal?BASE=CENSO_2017\&lang=esp

Kuo, B. C. (2014). Coping, acculturation, and psychological adaptation among migrants: a theoretical and empirical review and synthesis of the literature. Health
Psychology and Behavioral Medicine, 2(1), 16-33. doi: 10.1080/21642850.2013.843459

Lynn, R., \& Martin, T. (1995). National differences for thirtyseven nations in extraversion, neuroticism, psychoticism and economic, demographic and other correlates. Personality and Individual Differences, 19(3), 403-406. doi: 10.1016/0191-8869(95)00054-A

Martínez, J., \& Vono, D. (2005). Geografía migratoria intrarregional de América Latina y el Caribe al comienzo del siglo XXI. Revista de Geografia Norte Grande, 34, 39-52. Recuperado de https://www.redalyc.org/pdf/300/30003403. pdf

Mera, M. J., Martínez-Taboada, C., \& Costalat-Founeau, A. (2014). Dinámicas identitarias en procesos de transición psicosocial: adolescencia y migración. Un estudio de caso. Revista Migraciones Internacionales, 7(3), 221-248. doi: 10.17428/rmi.v7i26.676

Mera, M. J., Martínez-Zelaya, G., Bilbao, M. A., \& Garrido, A. (2017). Chilenos ante la inmigración: un estudio de las relaciones entre orientaciones de aculturación, percepción de amenaza y bienestar social en el Gran Concepción. Universitas Psychologica, 16(5), 1-14. doi: 10.11144/ Javeriana.upsy16-5.cier

Mooi, E., \& Sarstedt, M. (2011). A Concise Guide to Market Research. The Process, Data, and Methods Using IBM SPSS Statistics. Berlin: Springer.

Navas, M., Pumares, P., Sanchéz, J., García, M., Rojas, A., Cuadrado, I., Asensio, M., \& Fernández, J. (2004). Estrategias y actitudes de aculturación: la perspectiva de los inmigrantes y de los autóctonos en Almería. España: Dirección General de Coordinación de Políticas Migratorias, Consejería de Gobernación, Junta de Andalucía.

Ochoa E., Vicente N., \& Lozano, M. (2005). Síndromes depresivos en la población inmigrante. Revista Clínica Española, 205(3), 116-118. doi: 10.1157/13072968

Oyserman, D., Coon, H. M., \& Kemmelmeier, M. (2002). Rethinking Individualism and Collectivism: Evaluation of Theoretical assumptions and meta-analyses. Psychological Bulletin, 128(1), 3-72. doi: 10.1037/0033-2909.128.1.3

Ryff, C. (1989). Happiness is everything, or is it? Explorations on the meaning of psychological well-being. Journal of Personality and Social Psychology, 57(6), 1069-1081. doi: 10.1037/0022-3514.57.6.1069

Sam, D. L., \& Berry, J. W. (2010). Acculturation: when individuals and groups of different cultural backgrounds meet. Perspectives on Psychological Science, 5(4), 472-481. doi: 10.1177/1745691610373075

Silva, J., Urzúa, A., Caqueo-Urizar, A., Lufin, M., \& Irarrázaval, M. (2016). Bienestar psicológico y estrategias de aculturación en inmigrantes afrocolombianos en el norte de Chile. Interciencia: Revista de ciencia y tecnología de América, 41(12), 804-811. Recuperado de https://www.interciencia. net/wp-content/uploads/2017/10/804-CAQUEO-41-12.pdf 
Sirlopú, D., \& van Oudenhoven, J. (2013). Is multiculturalism a viable path in Chile? Intergroup and acculturative perspectives on Chilean society and Peruvian immigrants. International Journal of Intercultural Relations, 37(6), 739749. doi: 10.1016/j.ijintrel.2013.09.011

Stefoni, C. (2011). Perfil migratorio de Chile. Buenos Aires, Argentina: Organización Internacional para las Migraciones (OIM).

Smith, P. B., \& Bond, M. H. (1993). Social Psychology across cultures: Analysis and perspectives. New York: Harvester Wheatsheaf.

Tajfel, H., \& Turner, J. (1979). An integrative theory of intergroup conflict. En Worchel, S., \& Austin, W. G. (eds.), The Social psychology of intergroup relations (pp. 33-47). Monterey, C. A.: Brooks/Cole.

Torres, L., Driscoll, M. W., \& Voell, M. (2012). Discrimination, acculturation, acculturative stress, and Latino psychological distress: a moderated mediational model. Cultural Diversity y Ethnic Minority Psychology, 18(1), 17-25. doi: 10.1037/ a0026710

Triandis, H., Bontempo, R., Villareal, M., Asai, M., \& Lucca, N. (1988). Individualism and collectivism: Cross-cultural perspectives on self-ingroup relationships. Journal of Personality and Social Psychology, 54(2), 323-338. doi: 10.1037/0022-3514.54.2.323

Ugalde-Watson, K., Smith-Castro, V, Moreno-Salas, M., \& Rodriguez-García, J. M. (2011). Estructura, correlatos y predictores del estrés por aculturación. El caso de personas refugiadas colombianas en Costa Rica. Universitas Psychologica, 10(3), 759-774. Recuperado de https://revis tas.javeriana.edu.co/index.php/revPsycho/article/view/760

Urzúa, A., Basabe, N., Pizarro, J. J., \& Ferrer, R. (2017). Afrontamiento del estrés por aculturación: inmigrantes latinos en Chile. Universitas Psychologica, 16(5), 1-13. doi: 10.11144/Ja veriana.upsy16-5.aeai

Urzúa M., A., Boudon, S., \& Caqueo-Urízar, A. (2017). Salud Mental y Estrategias de Aculturación en inmigrantes colombianos y peruanos en el Norte de Chile. Acta Colombiana de Psicología, 20(1), 70-79. doi: 10.14718/ACP.2017.20.1.5

Urzúa, A., Heredia, O., \& Caqueo-Urízar, A. (2016). Salud mental y estrés por aculturación en inmigrantes sudamericanos en el norte de Chile. Revista Médica de Chile. 144(5), 563570. doi: 10.4067/S0034-98872016000500002

Urzúa, A., Sánchez, N., Paniagua, G., Igor, M. J., \& Castro, A. (2015). Bienestar Psicológico en inmigrantes peruanos y colombianos en el norte de Chile. En Bilbao, M .A, Oyanadel, J. C, y Páez, D. (eds), La felicidad de los chilenos: estudios sobre Bienestar (Vol. 1) (pp. 127-141). Santiago de Chile: RIL Editores.

Valenzuela, P., Riveros, K., Palomo, N., Araya, I., Campos, B., Salazar, C., \& Tavie, C. (2018). Integración laboral de los inmigrantes haitianos, dominicanos y colombianos en Santiago de Chile. Revista Antropologías del Sur, 1(2), 101121. doi: 10.25074/ads.v1i2.845

Van Leeuwen, N., Rodgers, R. F., Bui, E., Pirlot, G., \& Chabrol, H. (2014). Relations between acculturation orientations and antisocial behavior in adolescents and young adults from immigrant families. International Journal of Culture and Mental Health, 7(1), 68-82. doi: 10.1080/17542863.2012.699534

Van Leeuwen, N., Rodgers, R., Régner, R., \& Chabrol, H. (2010). The role of acculturation in suicidal ideation among secondgeneration immigrant adolescents in France. Transcultural Psychiatry, 47(5), 812-32. doi: 10.1177/1363461510382154

Ward, C., Bochner, S., \& Furnham, A. (2001). The psychology of culture shock. Londres: Routledge.

Williams, C. L., \& Berry, J. W. (1991). Primary prevention of acculturative stress among refugees: Application of psychological theory and practice. American Psychologist, 46(6), 632-641. doi: 10.1037/0003-066X.46.6.632

Yáñez, S., \& Cárdenas, M. (2010). Estrategias de aculturación, indicadores de salud mental y bienestar psicológico en un grupo de inmigrantes sudamericanos en Chile. Salud y Sociedad, 1(1), 51-70. doi: 10.22199/ S07187475.2010.0001.00006

Yoon, E., Chang, C. T., Kim S., Clawson, A., Cleary, S. E., Hansen, M., ... Gomes, A. (2013). A meta-analysis of acculturation/enculturation and mental health. Journal of Counseling Psychology, 60(1), 15-30. doi: 10.22199/ s07187475.2010.0001.00006 